\title{
From the Anderson Model on a Strip to the DMPK Equation and Random Matrix Theory
}

\author{
S. Bachmann · W. De Roeck
}

Received: 18 January 2010 / Accepted: 8 March 2010 / Published online: 2 April 2010

(C) The Author(s) 2010. This article is published with open access at Springerlink.com

\begin{abstract}
We study weakly disordered quantum wires whose width is large compared to the Fermi wavelength. It is conjectured that such wires display universal metallic behavior as long as their length is shorter than the localization length (which increases with the width). The random matrix theory that accounts for this behavior-the DMPK theory-rests on assumptions that are in general not satisfied by realistic microscopic models. Starting from the Anderson model on a strip, we show that a twofold scaling limit nevertheless allows to recover rigorously the fundaments of DMPK theory, thus opening a way to settle some conjectures on universal metallic behavior.
\end{abstract}

Keywords Random matrix theory $\cdot$ Disordered conductors $\cdot$ Metallic phase

\section{Introduction}

We discuss the heuristic DMPK (Dorokhov-Mello-Pereyra-Kumar) theory of disordered wires in Sect. 1, following to some extent the survey by Beenakker, [2]. Then in Sect. 2, we introduce our microscopic model, a variant of the Anderson model on a strip, and we state the rigorous results, Propositions 2 and 3. The last proposition establishes that the main assumption of DMPK theory - the replacement of the microscopic model by a random matrix ensemble-is satisfied in a certain scaling limit.

While we were finishing this manuscript, a related article [28] appeared on the archive. The results of [28] are very similar to ours, although the focus and motivation are different.

\footnotetext{
S. Bachmann

Department of Mathematics, University of California, Davis, CA 95616, USA

e-mail: svenbac@math.ucdavis.edu

W. De Roeck $(\varangle)$

Department of Mathematics, University of Helsinki, P.O. Box 68, 00014, Helsinki, Finland

e-mail: wojciech.deroeck@itf.fys.kuleuven.ac.be
} 
Fig. 1 A schematic picture of the disordered wire and its various parameters

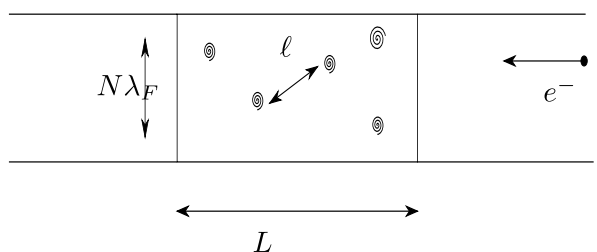

\subsection{Phenomenology of Disordered Wires}

Consider a wire of length $L$ and cross section $A$, made from a disordered material and let $\lambda \geq 0$ be a measure of the strength of the disorder. We assume that electrons are injected a fixed energy $E$ and are scattered coherently in the wire. Let $\ell$ be the mean free path ${ }^{1}$ of the electrons in the wire (hence $\ell$ depends on $\lambda$, namely $\ell \propto \lambda^{-2}$ ) and $\lambda_{F}$ the Fermi wavelength. We choose $\ell$ as the basic unit of length in which $L$ is measured and hence we define a reduced length

$$
s:=\frac{L}{\ell} .
$$

Physically, the Fermi wavelength $\lambda_{F}$ determines the number of channels $N$ that fit into the area $A$. Here, we shall rather take the dimensionless number $N$ as a measure of the cross section of the wire since, in our model, the number of channels will be naturally given. See Fig. 1 for a summary of the general setup.

If $N=1$, it is well-known that the electron gets localized with localization length $\approx \ell$ (hence $s \approx 1$ ). However, the localization length increases with $N$, and we can ask how the system behaves for $s \ll N$, before localization sets in. This regime turns out to be experimentally accessible and it is called the metallic, or diffusive, regime. One of the fascinating aspects of this regime is the phenomenon of universal conductance fluctuations (UCF). Let $g=g(s, N, \lambda)$ be the conductance of the wire, expressed in units of the conductance quantum $2 e^{2} / \hbar$. It is a random quantity due to the disorder. Its average satisfies Ohm's law as long as $s$ is smaller than the localization length $\sim N$ and it drops off exponentially once the localization length is exceeded. The rough behavior for large $N$ (see below for precise conjectures) is

$$
\mathbb{E}(g) \sim \begin{cases}N /(1+s)+O\left(N^{0}\right) & s \ll N(\text { Ohm's law) } \\ \exp \{-s / N\} & s>N \text { (localization) }\end{cases}
$$

where $\mathbb{E}(\cdot)$ indicates an average over the disorder. The UCF mean that

$$
\operatorname{Var}(g)=2 /(15 \beta), \quad \text { for } s \ll N
$$

has a fixed value, independent of the material or the wire length and width. The only parameter that remains is the symmetry index $\beta$ that refers to Dyson's symmetry classes.

It is important to note that these phenomena should emerge in a large $N$ limit only. On the other hand, $N$ cannot be too large because then we enter the regime of 2-dimensional localization (assuming that the wire has one transverse dimension). Below, we distill some mathematical conjectures from the physics literature. Keep in mind that $g$ is a random variable that depends on $s, N$ and $\lambda$, and that the disorder strength $\lambda$ enters in the definition of

\footnotetext{
${ }^{1}$ See [2] for a particular definition of the mean free path, which is necessary to avoid numerical constants in (1) and the Conjectures below.
} 
the unit of length $s$. Moreover, the conductance $g$ can in principle depend on the way disorder is brought in and on the energy $E$. Hence, in order to state the conjectures precisely, one should start from, for example, the microscopic model introduced in Sect. 2 (where $\beta=1$ ) and one should assume (possibly weakened versions of) Assumptions 1 and 2.

Conjecture 1 (Localization lengths) For $\lambda$ small enough but fixed

$$
\mathbb{E}(g)<\text { const } \cdot \mathrm{e}^{-\frac{s}{N}}, \quad \text { for all } s, N ;
$$

Conjecture 2 (Universal conductance fluctuations)

$$
\lim _{\substack{N \nearrow \infty \\ s / N=z \text { fixed }}} \lim _{\lambda \searrow 0} \operatorname{Var}(g)=2 /(15 \beta), \quad \text { for all } 0<z<1 ;
$$

Conjecture 3 (Ohm's law and weak localization correction)

$$
\lim _{\substack{N / \infty \\ s / N=z \text { fixed }}} \lim _{\lambda \searrow 0} \mathbb{E}(g)=\frac{1}{z}+\frac{1}{3}(1-2 / \beta), \quad \text { for all } 0<z<1,
$$

where the constant $\frac{1}{3}(1-2 / \beta)$, called the weak-localization correction, is yet another manifestation of universality.

At the time of writing, none of these conjectures is confirmed mathematically. In [23], a weakened version of Conjecture 1 was proven, with $N$ fixed and the range of admissible $\lambda$ 's shrinking as $N \nearrow \infty$. One should remark that Conjecture 1 differs from 2 and 3 in that the latter Conjectures cannot hold true without some scaling limit in which $\lambda \searrow 0$ i.e. $\ell \nearrow \infty$. Indeed, if we did not scale $\lambda$, then for large $s$ and $N$ one should recover the two-dimensional Anderson model, which is expected to be localized. Conjecture 1, however, makes sense even in the regime of two-dimensional localizations since it states only an upper bound on the localization length, linearly growing with the sample width $N$.

\subsection{Transfer Matrices and Conductance Properties}

A convenient macroscopic description of the wire is through its $2 N \times 2 N$ scattering matrix $S$. Heuristically, the $S$-matrix transforms incoming free waves into outgoing free waves. Let again $N$ be the number of channels and assume that the free dynamics is time-reversal symmetric; it follows that one can distinguish $N$ right-moving and $N$ left-moving free waves. Having this decomposition, the $S$-matrix acts as

$$
S\left(\begin{array}{c}
c_{R}^{\text {in }} \\
c_{L}^{\text {in }}
\end{array}\right)=\left(\begin{array}{c}
c_{L}^{\text {out }} \\
c_{R}^{\text {out }}
\end{array}\right), \quad S=\left(\begin{array}{cc}
r & t^{\prime} \\
t & r^{\prime}
\end{array}\right),
$$

where $c_{L}^{\text {in }}, c_{R}^{\text {out }}, c_{L}^{\text {out }}, c_{R}^{\text {in }}$ are $N$-dimensional vectors representing the amplitudes of the respective waves. The subscript $L / R$ indicates whether the wave is left/right moving respectively,

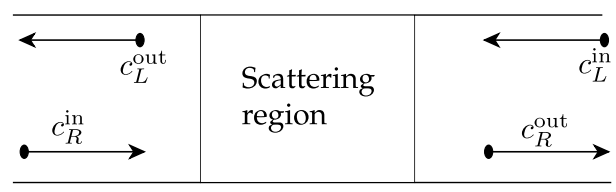


and the superscript in/out distinguishes incoming and outgoing waves. The $N \times N$ matrices $t, t^{\prime}$ and $r, r^{\prime}$ are usually called transmission and reflection matrices. Current conservation is equivalent to the unitarity of the $S$-matrix. Further, we assume that a particular basis has been chosen in $\mathbb{C}^{N}$ such that time-reversal is implemented by complex conjugation. If we assume that the scattering process is time-reversal invariant as well, then $S$ equals its transpose. In short, the two symmetries read

$$
S^{-1}=S^{*}, \quad \bar{S}=S^{*},
$$

where ${ }^{-}$denotes complex conjugation on each matrix element.

For our purposes, it is more convenient to encode the scattering data in the transfer matrix $M$, which transforms free waves on the left of the wire into free waves on the right. It acts as

$$
M\left(\begin{array}{c}
c_{R}^{\text {in }} \\
c_{L}^{\text {out }}
\end{array}\right)=\left(\begin{array}{c}
c_{R}^{\text {out }} \\
c_{L}^{\text {in }}
\end{array}\right) .
$$

Indeed, a left-moving wave on the right-hand side of the wire, and a right-moving wave on the left of the wire are incoming (they move from infinity towards the wire), whereas the two others are outgoing. Moreover, transfer matrices satisfy a simple multiplicative composition rule when two scatterers a put together in series. Finally, (8) and (6) imply simple algebraic relations between the matrix elements of $S$ and $M$, and the symmetries (7) translate into

$$
M^{*} \Sigma_{z} M=\Sigma_{z}, \quad \Sigma_{x} M \Sigma_{x}=\bar{M},
$$

where we employed the $2 N \times 2 N$ matrices

$$
\Sigma_{z}=\left(\begin{array}{cc}
1 & 0 \\
0 & -1
\end{array}\right), \quad \Sigma_{x}=\left(\begin{array}{ll}
0 & 1 \\
1 & 0
\end{array}\right)
$$

In other words,

$$
M=\left(\begin{array}{cc}
\alpha & \beta \\
\bar{\beta} & \bar{\alpha}
\end{array}\right),
$$

where the matrices $\alpha, \beta$ are constrained to satisfy

$$
\alpha^{*} \alpha-\bar{\beta}^{*} \bar{\beta}=1, \quad \alpha^{*} \beta-\bar{\beta}^{*} \bar{\alpha}=0 .
$$

In summary, a time-reversal invariant scattering process is described by a transfer matrix that belongs to the Lie group

$$
\mathcal{G}:=\left\{M \in G L(2 N) \mid M^{*} \Sigma_{z} M=\Sigma_{z} \text { and } \Sigma_{x} M \Sigma_{x}=\bar{M}\right\} .
$$

It follows from polar decomposition of the reflection and transmission matrices (or equivalently of $\alpha, \beta$ above) that any element of $\mathcal{G}$ can be written as

$$
M=\left(\begin{array}{cc}
U & 0 \\
0 & \bar{U}
\end{array}\right)\left(\begin{array}{cc}
\sqrt{T^{-1}} & \sqrt{T^{-1}-1} \\
\sqrt{T^{-1}-1} & \sqrt{T^{-1}}
\end{array}\right)\left(\begin{array}{cc}
V & 0 \\
0 & \bar{V}
\end{array}\right)
$$

where $U, V$ are unitary matrices and $T$ is a diagonal matrix whose entries are the eigenvalues of $t^{*} t$, and $t$ is the $N \times N$ transmission matrix appearing in $S$, see (6). Note that the matrices $U, V$ are not unambiguously determined because of the invariance of (12) under

$$
U \mapsto U A, \quad V \mapsto A V,
$$


where $A$ is a diagonal matrix with the diagonal consisting of -1 and +1 's. The group theoretical aspects of transfer matrices are discussed in detail in [18, 24].

We mentioned earlier that the conductance properties of a wire with transfer matrix $M$ can be read off from the eigenvalues of $T$. More precisely, the conductance can be defined via the Landauer-Büttiker formula as

$$
g:=\sum_{i=1}^{N} T^{i}=\operatorname{Tr} T=\operatorname{Tr} t^{*} t
$$

where $T^{i}$ are the eigenvalues of $T$. Of course, it is also possible to define the conductance through a physical setup, and then to prove that it is given by (14), but this will not concern us here.

\subsection{The DMPK-Theory of Disordered Quantum Wires}

\subsubsection{Stochastic Differential Equation for Transfer Matrics}

The basic postulate of the DMPK theory states that the random transfer matrix $\mathcal{M}(s)$ corresponding to wire length $s$ (in units of the mean free path) satisfies an Ito stochastic differential equation

$$
d \mathcal{M}(s)=d \mathcal{L}(s) \mathcal{M}(s), \quad \mathcal{M}(0)=1,
$$

where $\mathcal{L}(s)$ is a matrix-valued Brownian motion, and $d \mathcal{L}(s)$ is independent of $\mathcal{M}(s)$ (in mathematical terms: the process $\mathcal{M}(s)$ is adapted to the filtration generated by $\mathcal{L}(s)$, see e.g. [20] for details). Equation (15) is an immediate consequence of the composition rule for transfer matrices

$$
\mathcal{M}(s+d s)=(1+d \mathcal{L}(s)) \mathcal{M}(s)
$$

where $1+d \mathcal{L}(s)$ is the transfer matrix for the infinitesimal piece of wire of length $d s$. In practice, one assumes that $d s$ is large compared with the mean free path $\ell$, but small enough such for the transfer matrix $1+d \mathcal{L}(s)$ to be close to the identity. We immediately point out that this the latter requirement is not canonical. This is due to the fact that we use conventions such that, at zero disorder, the transfer matrix equals $1 .^{2}$ In our mathematical treatment of a microscopic model, this will be assured by expressing the transfer matrix in an interaction picture' where the fast oscillations due to the nonrandom ballistic evolution are subtracted.

Current conservation and time-reversal invariance, (9), restrict $d \mathcal{L}(s)$ to satisfy

$$
\left\{\begin{array}{l}
d \mathcal{L}^{*}(s) \Sigma_{z}+\Sigma_{z} d \mathcal{L}(s)+d \mathcal{L}^{*}(s) \Sigma_{z} d \mathcal{L}(s)=0 \\
d \mathcal{L}^{*}(s) \Sigma_{x}+\Sigma_{x} d \mathcal{L}^{*}(s)=0
\end{array}\right.
$$

as can easily be checked by the Ito calculus. In the upcoming Sect. 1.3.2, we shall postulate the law of $d \mathcal{L}(s)$ as it is derived in the physics literature from maximal entropy considerations. However, for the sake of the geometrically minded reader, we remark that Hüffmann [18] derives the form of (15) by demanding that the diffusion is generated by the LaplaceBeltrami operator on a certain symmetric space (note that there is no canonical LaplaceBeltrami operator on $\mathcal{G}$ as it is a noncompact Lie group).

\footnotetext{
${ }^{2}$ In the terminology of [2], we assume the 'equivalent channel' assumption rather than the 'isotropy' assumption.
} 


\subsubsection{The "Maximum Entropy” Assumption}

With (15) at hand, the major assumption of the DMPK theory is the 'isotropy', or 'maximal entropy' (MEA) assumption which states that $d \mathcal{L}(s)$ is drawn from an ensemble of maximal entropy, constrained on the total scattering, $\operatorname{Tr}(1-T(d \mathcal{L}(s)))$, due to $d \mathcal{L}(s)$. We do not go into this derivation and we merely state its conclusion, namely that $\mathcal{L}(s)$ is the following matrix-valued process,

$$
\mathcal{L}(s):=\left(\begin{array}{cc}
\mathfrak{a}(s) & \mathfrak{b}(s) \\
\overline{\mathfrak{b}}(s) & \overline{\mathfrak{a}}(s)
\end{array}\right)
$$

where

$$
\begin{aligned}
& \mathfrak{a}_{i j}(s)= \begin{cases}1 / \sqrt{2 N} \cdot\left(B_{i j}^{R}(s)+\mathrm{i} B_{i j}^{I}(s)\right) & 1 \leq i<j \leq N, \\
\mathrm{i} / \sqrt{N} \cdot B_{i i}^{I}(s) & i=j, \\
-\overline{\mathfrak{a}}_{j i}(s) & \text { otherwise, }\end{cases} \\
& \mathfrak{b}_{i j}(s)= \begin{cases}1 / \sqrt{2 N} \cdot\left(\widetilde{B}_{i j}^{R}(s)+\mathrm{i} \widetilde{B}_{i j}^{I}(s)\right) & 1 \leq i \leq j \leq N, \\
\mathfrak{b}_{j i}(s) & \text { otherwise, }\end{cases}
\end{aligned}
$$

and $B_{i j}^{R}(s), \widetilde{B}_{i j}^{I}(s), B_{i j}^{I}(s), \widetilde{B}_{i j}^{R}(s)$ are independent standard Brownian motions for $1 \leq i \leq$ $j \leq N$. We note that by construction

$$
\mathfrak{a}=-\mathfrak{a}^{*} \quad \text { and } \quad \mathfrak{b}^{*}=\overline{\mathfrak{b}},
$$

and we have the following invariance property: For any unitary matrix $W$,

$$
\begin{aligned}
W \mathfrak{a} W^{*} & \sim \mathfrak{a}, \\
W \mathfrak{b} \bar{W}^{*} & \sim \mathfrak{b},
\end{aligned}
$$

where $\sim$ denotes that the random variables have the same law. In particular, this invariance property means that we need not specify the basis in (17)-(18). Hence, ia is GUEdistributed, as one could also have noticed from the explicit expression (17). As regards $d \mathcal{L}(s)$, our choice for $\mathfrak{a}$ and $\mathfrak{b}$ implies that

$$
\mathcal{W} d \mathcal{L}(s) \mathcal{W}^{*} \sim d \mathcal{L}(s)
$$

where

$$
\mathcal{W}:=\left(\begin{array}{cc}
W & 0 \\
0 & \bar{W}
\end{array}\right) \quad \text { for any unitary } W .
$$

This expresses the intuition that the disorder in the wire is not 'biased' towards any specific channel basis. In conclusion, the SDE (15), together with the isotropy assumption encoded in the law defined by $(21,22)$, defines a stochastic flow in the group $\mathcal{G}$ (MEA-flow).

Assume that we aim to calculate the transmission eigenvalues $T_{i}(s)$ associated to the transfer matrix $\mathcal{M}(s)$. This can be done by Ito calculus, since the eigenvalues $T_{i}(s)$ are Ito processes that are functions of $\mathcal{M}(s)$. We postpone the explicit result of this calculation to the next section. First, we explore the main consequence of the symmetry (21) of $d \mathcal{L}(s)$. 
We write $T(s)=T(\mathcal{M}(s))$ for the diagonal matrix of transmission eigenvalues associated to $\mathcal{M}(s)$ by the decomposition (12). Likewise, we write $U(s), V(s)$, remembering that they are not uniquely determined by $\mathcal{M}(s)$. We also abbreviate

$$
\begin{aligned}
\mathcal{U}(s) & :=\left(\begin{array}{cc}
U(s) & 0 \\
0 & \bar{U}(s)
\end{array}\right), \\
\mathcal{V}(s) & :=\left(\begin{array}{cc}
V(s) & 0 \\
0 & \bar{V}(s)
\end{array}\right), \\
\mathcal{N}(s) & :=\left(\begin{array}{cc}
\sqrt{T^{-1}(s)} & \sqrt{T^{-1}(s)-1} \\
\sqrt{T^{-1}(s)-1} & \sqrt{T^{-1}(s)}
\end{array}\right) .
\end{aligned}
$$

Let $\mathcal{W}$ be as in (22) with $W$ Haar-distributed in the unitary group. Then, formally,

$$
\begin{aligned}
T(\mathcal{M}(s+d s)) & =T((1+d \mathcal{L}(s)) \mathcal{U}(s) \mathcal{N}(s) \mathcal{V}(s)) \\
& \sim T\left(\mathcal{W}(1+d \mathcal{L}(s)) \mathcal{W}^{*} \mathcal{U}(s) \mathcal{N}(s) \mathcal{V}(s)\right) \\
& \sim T\left(\mathcal{U} \mathcal{W}(1+d \mathcal{L}(s)) \mathcal{W}^{*} \mathcal{N}(s)\right) \\
& \sim T((1+d \mathcal{L}(s)) \mathcal{N}(s)),
\end{aligned}
$$

where we used (21), observed that $\mathcal{W}^{*}(s) \mathcal{U}(s) \sim \mathcal{W}^{*}(s)$ since the unitary operators $W$ in $\mathcal{W}(s)$ are Haar-distributed, and that the diagonal matrix $T(s)$ is not affected by the unitaries in the decomposition (12). Importantly, this computation shows that $T(s+d s)$ depends on $\mathcal{M}(s)$ only via $T(s)$ (because it only depends on $\mathcal{N}(s)$ ), whereas in general, the eigenvalues at $s+d s$ ought to depend on both the eigenvalues and eigenvectors at $s$.

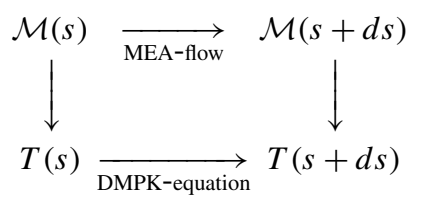

This phenomenon, represented by the commuting diagram (23), is known in statistical mechanics as the autonomous evolution of a (set of) macroscopic degree(s) of freedom. Of course, it is rather well known in random matrix theory as well, as it appears in Dyson's Brownian motion, see e.g. [8, 17]. Another conclusion is that, for the sake of calculating $T(s+d s)$ from $T(s)=T(\mathcal{M}(s))$, we could as well have assumed that the unitary $U(s)$ in $\mathcal{U}(s)$ is Haar-distributed. We can also turn the previous argument around, to obtain the following statement

If, for all $s$, the matrix $U(s)$ that appears in the polar decomposition (12) of $\mathcal{M}(s)$ is Haar distributed in the unitary group and independent of $T(\mathcal{M}(s))$, then $T(\mathcal{M}(s+d s))$ can be calculated form $T(\mathcal{M}(s))$ as if $d \mathcal{L}(s)$ were distributed as in (16).

This observation suggests a heuristic explanation as to why the DMPK equation can still be a good approximation when $d \mathcal{L}(s)$ is not distributed as in (16). By using the 'concentration of measure' property on the unitary group, see e.g. [17], we understand that the set of $U(s)$ such that the dependence of $T(\mathcal{M}(s+d s))$ on $T(\mathcal{M}(s))$ deviates significantly from that predicted by the DMPK equation, has an exponentially shrinking size w.r.t. the Haar measure, as $N \nearrow \infty$. 


\subsubsection{The DMPK Equation}

In the previous section, we already anticipated the fact that the evolution of the transmission eigenvalues $T_{k}(s)$ is autonomous. We now give the precise evolution equation for them.

Since $T_{k}(s)=T_{k}(\mathcal{M}(s))$ is a function of $\mathcal{M}(s)$, the law of its process can be calculated by Ito's fomula:

$$
d T_{k}(s)=\sum_{i j} \frac{\partial T_{k}}{\partial \mathcal{M}_{i j}}(s) d \mathcal{M}_{i j}(s)+\frac{1}{2} \sum_{i j, i^{\prime}, j^{\prime}} \frac{\partial^{2} T_{k}}{\partial \mathcal{M}_{i^{\prime} j^{\prime}} \partial \mathcal{M}_{i j}}(s) d \mathcal{M}_{i^{\prime} j^{\prime}}(s) d \mathcal{M}_{i j}(s) .
$$

The derivatives $\frac{\partial T_{k}}{\partial \mathcal{M}_{i j}}, \frac{\partial^{2} T_{k}}{\partial \mathcal{M}_{i^{\prime} j^{\prime}} \partial \mathcal{M}_{i j}}$ can be computed by spectral perturbation theory under the assumption that the diagonal matrix $T(s)$ is non-degenerate, i.e. all transmission eigenvalues are simple.

The formal result is the DMPK equation (discovered by $[6,19]$ )

$$
d T_{k}(s)=v_{k}(T(s)) d s+D_{k}(T(s)) d B_{k}(s)
$$

where $B_{k}(s), k=1, \ldots, N$ are independent standard Brownian motions and the drift $^{3}$ $v_{k}(T(s))$ and diffusion constant $D_{k}(T(s))$ are given by (we keep the parameter $\beta$ to make the comparison with the literature simpler, in our case $\beta=1$ )

$$
\begin{aligned}
v_{k} & =-T_{k}+\frac{2 T_{k}}{\beta N+2-\beta} \times\left(1-T_{k}+\beta / 2 \sum_{j \neq k} \frac{T_{k}+T_{j}-2 T_{k} T_{j}}{T_{k}-T_{j}}\right), \\
D_{k} & =4 \frac{T_{k}^{2}\left(1-T_{k}\right)}{\beta N+2-\beta} .
\end{aligned}
$$

We refer to [2] for a more detailed account of this straightforward, but somewhat lengthy, calculation. We also note that the term 'DMPK equation' usually refers to the Fokker-Planck equation associated with the SDE (24). The Brownian motions $B_{k}(s)$ in (24) originate from linear combinations of the Brownian motions in (17). We call the result (24) formal since we have not established that the equation admits a solution for all times, i.e. we have not excluded that two or more eigenvalues can collide, see [17] on a possible strategy to do this.

The basic picture concerning (24) is that, as $s$ grows, all $T_{k}(s)$ are driven to 0 by the term $-T_{k}$ in the drift (note that $0 \leq T_{k} \leq 1$ ). However, due to the repulsion in the term containing $\left(T_{k}-T_{j}\right)^{-1}$, the $T_{k}$ 's keep a distance of order $1 / N$. Finally, for small values of $T_{k}, T_{j}$, the repulsion vanishes and the $T_{k}$ 's pile up at 0 (in the localized regime, they are all very close to 0 ). The most interesting question is now whether (24) reproduces the phenomena discussed in Sect. 1.1. In other words,

Does the random variable $g$ satisfy the conjectures listed in Sect. 1.1 if one defines $g=\sum_{i} T_{i}$ with $T_{i}$ the solutions of (24)?

On the heuristic level, the answer is clearly "yes" for all 3 Conjectures, see e.g. [2] for an expansion scheme for large $N$. On the rigorous level however, the situation is not so clear

\footnotetext{
${ }^{3}$ We follow the literature on the subject in calling $v_{k}$ and $D_{k}$ the drift and the diffusion. One could however argue that this is a confusing convention, since absence of drift, $v_{k}=0$ does not render the process timereversal invariant, cfr. difference between Ito and Stratonovich conventions.
} 
(at least to us). For Conjecture 1, the techniques of [23], developed for a harder problem, are sufficiently strong to settle the question, see e.g. [22]. The perspectives seem to be best for $\beta=2$ where the DMPK equation can be solved exactly [3] in terms of toroidal functions. It is plausible that in this case, one can prove all Conjectures listed, but we do not know any reference where this is actually done. For $\beta=1,4$, the DMPK equation was solved in $[4,18,30]$, but in those cases, the solution is less explicit.

\section{The Microscopic Model: Anderson Model an a Strip}

\subsection{The Hamiltonian for a Disordered Wire}

Our system is an infinitely extended wire of width $N$, which we model by the Hilbert space $l^{2}(\mathbb{Z} \times\{1, \ldots, N\}) \cong l^{2}(\mathbb{Z}) \otimes \mathbb{C}^{N}$. We consider the motion of a single electron through the wire. It is governed by a Hamiltonian consisting of a ballistic part, and a disorder term, $H_{\lambda}=H_{\text {bal }}+\lambda V$, where $\lambda$ governs the strength of the disorder. The ballistic Hamiltonian of the system is given by a longitudinal $H_{\|}$, acting only on $l^{2}(\mathbb{Z})$, and a transversal $H_{\perp}$, acting only on $\mathbb{C}^{N}$;

$$
H_{\text {bal }}=H_{\|} \otimes 1+1 \otimes H_{\perp}
$$

and we will slightly abuse the notation by writing

$$
H_{\|}=H_{\|} \otimes 1, \quad H_{\perp}=1 \otimes H_{\perp} .
$$

We choose $H_{\|}$to be the standard lattice Laplacian on $l^{2}(\mathbb{Z})$, with kernel

$$
H_{\|}\left(x, x^{\prime}\right)=\delta_{x, x^{\prime}-1}+\delta_{x, x^{\prime}+1}, \quad x, x^{\prime} \in \mathbb{Z} .
$$

The disorder is modeled by a random on-site potential, which we assume to be located only on the sites with longitudinal coordinate between 1 and $L$, as shown schematically in Fig. 2. Hence, $L$ is the length of the disordered part of the wire and

$$
V:=\sum_{\substack{x=1, \ldots, L \\ z=1, \ldots, N}} v_{x, z} 1_{(x, z)},
$$

with $1_{(x, z)}$ the one-dimensional projector on the site $(x, z) \in \mathbb{Z} \times\{1, \ldots, N\}$, and $v_{x, z}$ are i.i.d. random variables with mean 0 and variance 1 . We also assume that the third, fourth and fifth moments of $v_{x, z}$ exist, i.e.

$$
\mathbb{E}(v)=0, \quad \mathbb{E}\left(v^{2}\right)=1, \quad \mathbb{E}\left(v^{3}\right), \mathbb{E}\left(v^{4}\right), \mathbb{E}\left(v^{5}\right)<\infty .
$$

Fig. 2 The microscopic model

I.i.d. random variables $v_{x, z}$

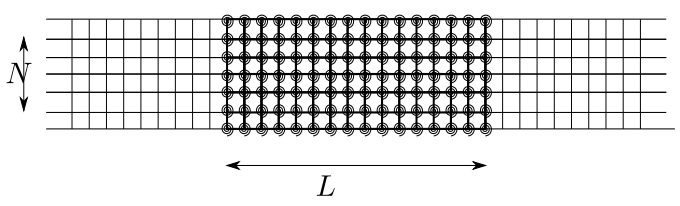


The operator $H_{\perp} \in \mathcal{B}\left(\mathbb{C}^{N}\right)$ can for some purposes by chosen largely at will (though we will always impose Assumptions 1 and 2 below) but for our main result we will require that $H_{\perp}$ is the transverse Laplacian with periodic boundary conditions and with a magnetic field that breaks the chiral symmetry (to be introduced precisely later). The eigenvectors of $H_{\perp}$, denoted by $\psi_{\mu}, \mu=1, \ldots, N$ fix a basis in $\mathbb{C}^{N}$,

$$
H_{\perp} \psi_{\mu}=E_{\perp}(\mu) \psi_{\mu}, \quad \mu=1, \ldots, N
$$

with corresponding energies $E_{\perp}(\mu)$. The index $\mu$ is hence a free channel-index.

After diagonalizing the free transverse dynamics, we define

$$
E_{\|}:=O^{*}\left(E-H_{\perp}\right) O=\operatorname{diag}\left(E_{\|}(1), \ldots, E_{\|}(N)\right),
$$

where the eigenvalue $E_{\|}(\mu)=E-E_{\perp}(\mu)$ represents the longitudinal energy in the $\mu$ th channel. The obvious condition for a channel to be conducting is that $E_{\|}(\mu)$ lies in the spectrum of $H_{\|}$:

Assumption 1 (Elliptic channels) Recall that the spectrum of $H_{\|}$is the interval $[-2,2]$. We demand

$$
E_{\|}(\mu) \in \operatorname{int}\left(\operatorname{spec} H_{\|}\right) \quad \text { for all } \mu=1, \ldots, N \text {. }
$$

This assumption does for example exclude a $H_{\|}$describing a barrier that would stop the waves, even in the absence of disorder.

For reasons that will become clear later (see Sect. 3.2), we need to make sure that the system has no accidental symmetries. A first requirement would be that the operator $H_{\perp}$ is nondegenerate, but we demand a stronger condition, namely:

Assumption 2 (No degenerate level spacings) Let $H_{\perp}$ be nondegenerate, i.e. $E_{\|}(\mu) \neq$ $E_{\|}(v)$ for $\mu \neq v$. Let $\theta_{\mu}=\theta\left(E_{\|}(\mu)\right)$ be defined by

$$
2 \cos \theta_{\mu}=E_{\|}(\mu), \quad 0 \leq \theta_{\mu}<\pi .
$$

Let $\mu_{i} \in\{1, \ldots, N\}$ for $i=1,2,3,4$. Then the equality

$$
\sum_{i=1}^{4} q_{i} \theta_{\mu_{i}}=0, \quad \text { where } q_{i} \in\{-1,1\}
$$

can only hold (up to a permutation of the indices $\{1,2,3,4\})$ if

$$
\mu_{1}=\mu_{2} \quad \text { and } \quad \mu_{3}=\mu_{4}, \quad \text { and } \quad q_{1} q_{2}=q_{3} q_{4}=-1 .
$$

As $E_{\|}(\mu)$ is the energy available for longitudinal propagation in the $\mu$ th-channel, $\theta_{\mu}$ corresponds the absolute value of the longitudinal momentum in each channel. Equation (28) is indeed nothing else than the dispersion relation for $H_{\|}$.

\subsection{Transfer Matrix}

The setup described above suggests to study the (random) scattering matrix $S=S(\lambda, L, N)$ of the wire, since the motion is ballistic outside a compact region. 
Abstractly, the scattering operator is obtained by comparing the full dynamics to the free one generated by the ballistic Hamiltonian (25),

$$
S=s-\lim _{t_{ \pm} \rightarrow \pm \infty} U_{0}\left(-t_{-}\right) U\left(t_{-}-t_{+}\right) U_{0}\left(t_{+}\right)
$$

where $U(t)=\exp \left(-H_{\lambda} t\right)$ and $U_{0}(t)=\exp \left(-H_{\mathrm{bal}} t\right)$. The question of asymptotic completeness, namely the existence of the limits and the unitarity of the scattering operator, is readily solved by noting that the perturbation $\lambda V$ is of finite rank and invoquing the KatoRosenblum theorem [21]. Finally in the fiber decomposition $\mathcal{H}=\int^{\oplus} \mathcal{H}_{E} d E$, the scattering operator is described by a family of $2 N \times 2 N$ scattering matrices $S_{E}$.

If Assumption 1 is satisfied, the fiber spaces $\mathcal{H}_{E}$ are isomorphic to $\mathbb{C}^{2 N} \cong \mathbb{C}_{L}^{N} \oplus \mathbb{C}_{R}^{N}$, which physically corresponds to a left and a right moving sector. Once the incoming energy is fixed and the index $E$ dropped, this definition coincides with the more physical one, (6), based on transmitted and reflected plane waves, see e.g. [1]. In that representation, current conservation is equivalent to the unitarity of the scattering matrix, which is ensured by asymptotic completeness. If, in addition, the matrix $S$ is written down in the eigenbasis $\left\{\psi_{\mu} \oplus 0,0 \oplus \overline{\psi_{\mu}}\right\}_{\mu=1}^{N}$ defined in (27), time-reversal invariance indeed corresponds to $S^{*}=\bar{S}$ with * the complex conjugation on each matrix entry.

As noted in Sect. 1, we shall work in the following with the equivalent transfer matrix $M=M(\lambda, L, N)$. In order to construct it explicitly, we first introduce the family of matrices $T_{x}^{\lambda}, x=1, \ldots, L$, which can be constructed without explicit reference to scattering, and this is the way this setup is usually presented in the mathematical literature. Define the $2 \mathrm{~N} \times 2 \mathrm{~N}$ matrix

$$
T_{x}^{\lambda}=\left(\begin{array}{cc}
E_{\|} & -1_{N} \\
1_{N} & 0
\end{array}\right)=\left(O^{*} \otimes 1_{2}\right)\left(\begin{array}{cc}
E-H_{x x} & -1_{N} \\
1_{N} & 0
\end{array}\right)\left(O \otimes 1_{2}\right),
$$

where $H_{x x}:=1_{x} H 1_{x}=H_{\perp}+\lambda V_{x}$ and $V_{x}=\sum_{z=1}^{N} v_{x, z} 1_{(x, z)}$. The key property of $T_{x}^{\lambda}$ is that, if $\Psi=\left(\Psi_{x}\right)_{x \in \mathbb{Z}}, \Psi_{x} \in \mathbb{C}^{N}$ is a (not necessarily normalizable) solution of the eigenvalue equation $H_{\lambda} \Psi=E \Psi$, then

$$
\left(\begin{array}{c}
\Psi_{x+1} \\
\Psi_{x}
\end{array}\right)=T_{x}^{\lambda}\left(\begin{array}{c}
\Psi_{x} \\
\Psi_{x-1}
\end{array}\right) .
$$

Of course, this property is preserved under multiplication, i.e.

$$
\left(\begin{array}{c}
\Psi_{L+1} \\
\Psi_{L}
\end{array}\right)=T^{\lambda}(L)\left(\begin{array}{c}
\Psi_{1} \\
\Psi_{0}
\end{array}\right), \quad T^{\lambda}(L):=T_{L}^{\lambda} T_{L-1}^{\lambda} \cdots T_{1}^{\lambda} .
$$

To describe our results in the most natural way, it is convenient to express the transfer matrix in the basis of free in- and out-states, i.e. in left- and right-moving waves in the different channels $\mu$, as was done in Sects. 1.2 and 1.3. Therefore, we introduce the transformation $\Upsilon$ mapping the transfer matrix $T_{x}^{\lambda}$ in the position representation to the more physical transfer matrix

$$
M_{x}^{\lambda}=\Upsilon^{-1} T_{x}^{\lambda} \Upsilon, \quad x=1, \ldots, L .
$$

For $N=1$, this change of basis amounts to

$$
\left(\begin{array}{c}
\mathrm{e}^{\mathrm{i} \theta(\varepsilon)} \\
1
\end{array}\right)=\Upsilon\left(\begin{array}{l}
1 \\
0
\end{array}\right), \quad\left(\begin{array}{c}
\mathrm{e}^{-\mathrm{i} \theta(\varepsilon)} \\
1
\end{array}\right)=\Upsilon\left(\begin{array}{l}
0 \\
1
\end{array}\right)
$$


(the vectors on the left should be thought of as incoming and outgoing 'plane waves') and for $N>1$, we have $N$ copies of this transformation, with $\varepsilon=E_{\|}(\mu)$ now channel-dependent, see (45) for an explicit expression. Note that although it represents a mere basis transformation, $\Upsilon$ is not unitary since the bases are not orthogonal.

In that representation, the transfer matrix $M_{x}^{\lambda}$ is diagonal in the absence of disorder, namely

$$
M^{0}=M_{x}^{0}=\left(\begin{array}{cc}
\exp \left(\mathrm{i} \theta\left(E_{\|}\right)\right) & 0 \\
0 & \exp \left(-\mathrm{i} \theta\left(E_{\|}\right)\right)
\end{array}\right),
$$

and corresponds indeed to its heuristic definition. In the following lemma, we summarize the precise relation between the microscopic model and the matrices $M_{x}^{\lambda}$. This is just a mathematical confirmation of the discussion in Sect. 1.

Lemma 1 The transfer matrices $M_{x}^{\lambda}$, defined through (30) and (31) belong to the group $\mathcal{G}$, see (11). They are related to the scattering matrix, as defined in (29), by the relations given in Sect. 1.2.

Note further that the transfer matrices constructed in this section represent the influence of one lattice site. The transfer matrices for longer parts of the wire are introduced below.

\subsection{Results}

\subsubsection{Scaling Limit}

Naively, we interpret the DMPK theory as suggesting that the transfer matrix for the wire with rescaled length $s$,

$$
M^{\lambda}\left(\left\lfloor\lambda^{-2} s\right\rfloor\right):=M_{\lfloor\lambda-2 s\rfloor}^{\lambda} \ldots M_{1}^{\lambda},
$$

(where $\lfloor a\rfloor$ stands for the largest integer not greater than $a$ ) converges to the solution of the differential equation (15) as $\lambda \searrow 0$. This cannot be correct as such. First of all, $M^{\lambda}\left(\left\lfloor\lambda^{-2} s\right\rfloor\right)$ contains some rapidly oscillating terms (as functions of $\lambda^{-2} s$ ) whose phase cannot be welldefined as a function of $s$. Recalling that the transfer matrix in the absence of disorder $(V=0)$ is of the form

$$
M^{0}\left(\left\lfloor\lambda^{-2} s\right\rfloor\right)=\left(\begin{array}{cc}
\exp \left(\mathrm{i}\left\lfloor\lambda^{-2} s\right\rfloor \theta\left(E_{\|}\right)\right) & 0 \\
0 & \exp \left(-\mathrm{i}\left\lfloor\lambda^{-2} s\right\rfloor \theta\left(E_{\|}\right)\right)
\end{array}\right),
$$

we introduce a first modification to our convergence conjecture. We multiply by the inverse of this free scatting matrix to subtract the fast oscillations, i.e. we consider

$$
A^{\lambda}\left(\left\lfloor\lambda^{-2} s\right\rfloor\right):=\left(M^{0}\left(\left\lfloor\lambda^{-2} s\right\rfloor\right)\right)^{-1} M^{\lambda}\left(\left\lfloor\lambda^{-2} s\right\rfloor\right)
$$

which is 1 at $V=0$. The new conjecture is that $A^{\lambda}\left(\left\lfloor\lambda^{-2} s\right\rfloor\right)$ converges to the solution of the DMPK equation. This weakened form is still not correct without further assumptions although we believe that it is 'essentially correct' (as we shall explain below). Actually, $A^{\lambda}\left(\left\lfloor\lambda^{-2} s\right\rfloor\right)$ converges to the solution of a SDE that differs from the DMPK equation because it has a lower symmetry. 
Proposition 2 If Assumptions 1 and 2 hold, then $A^{\lambda}\left(\left\lfloor\lambda^{-2} s\right\rfloor\right)$ converges in distribution, ${ }^{4}$

$$
A^{\lambda}\left(\left\lfloor\lambda^{-2} s\right\rfloor\right) \underset{\text { distr }}{\stackrel{\lambda \succeq 0}{\longrightarrow}} \mathcal{A}(s) \text { for all } s \text {. }
$$

The process $\mathcal{A}(\cdot)$ is the strong solution of the $S D E$

$$
d \mathcal{A}(s)=d \mathcal{Z}(s) \mathcal{A}(s), \quad \mathcal{A}(0)=1
$$

where

$$
\mathcal{Z}(s)=\left(\begin{array}{ll}
\mathfrak{a}^{\prime}(s) & \mathfrak{b}^{\prime}(s) \\
\overline{\mathfrak{b}}^{\prime}(s) & \overline{\mathfrak{a}}^{\prime}(s)
\end{array}\right) .
$$

The matrix-valued Brownian motions $\mathfrak{a}^{\prime}(s), \mathfrak{b}^{\prime}(s)$ are defined as

$$
\begin{array}{r}
\mathfrak{a}_{\mu \nu}^{\prime}(s)=\sigma_{\mu \nu} \begin{cases}1 / \sqrt{2 N} \cdot\left(B_{\mu \nu}^{R}(s)+\mathrm{i} B_{\mu \nu}^{I}(s)\right) & 1 \leq i<j \leq N, \\
\mathrm{i} / \sqrt{N} \cdot B_{\mu}^{I}(s) & i=j, \\
-\overline{\mathfrak{a}_{v \mu}^{\prime}} & \text { otherwise, }\end{cases} \\
\mathfrak{b}_{\mu \nu}^{\prime}(s)=\sigma_{\mu \nu} \begin{cases}1 / \sqrt{2 N} \cdot\left(\widetilde{B}_{\mu \nu}^{R}(s)+\mathrm{i} \widetilde{B}_{\mu \nu}^{I}(s)\right) & 1 \leq i \leq j \leq N, \\
\mathfrak{b}_{v \mu}^{\prime}(s) & \text { otherwise. }\end{cases}
\end{array}
$$

(the various standard Brownian motions appearing here are similar to those of $(17,18)$ ) with

$$
\left(\sigma_{\mu \nu}\right)^{2}=\frac{N \mathbb{E}\left|\widetilde{V}_{\mu \nu}\right|^{2}}{4 \cdot\left|\sin \theta_{\mu} \sin \theta_{\nu}\right|},
$$

where $\mathbb{E}\left|\widetilde{V}_{\mu \nu}\right|^{2}$ denote the covariances of the $N \times N$ matrix $\widetilde{V}=O^{*} V O$ of the random potentials in the channel basis and the momenta $\theta_{\mu}$ were defined in Assumption 2.

We note immediately that the SDE (34) differs from the corresponding SDE (15) in DMPK theory by the factors $\sigma_{\mu \nu}$ that do depend on the channels. For the same reason, the definitions (35) and (36) are truly basis-dependent ( $\mu, v$ index the channels). This remains true even if the Hamiltonian $H_{\perp}$ is chosen in the most isotropic way, as outlined below.

Let $H_{\perp}$ on $\mathbb{C}^{N}$ be given by the kernel

$$
H_{\perp}\left(z, z^{\prime}\right):=h_{\perp}\left(\mathrm{e}^{\mathrm{i} \gamma} \delta_{z, z^{\prime}-1}+\mathrm{e}^{-\mathrm{i} \gamma} \delta_{z, z^{\prime}+1}\right), \quad z, z^{\prime} \in \mathbb{Z} / N \mathbb{Z}
$$

(with $0<\gamma<2 \pi / N$ and $h_{\perp}>0$ ) which corresponds to a cylindrical wire permeated by a magnetic field $\gamma$ along its symmetry axis. Note that we cannot choose $\gamma=2 \pi k / N, k \in \mathbb{Z}$, without violating Assumption 2. In those cases, $H_{\perp}$ corresponds to the pure Laplacian on a discrete torus, which has a chiral symmetry and hence twice degenerate energy levels. The choice (38) also forbids the injection energy $E$ to lie at the band center. Indeed, since the spectrum of $H_{\perp}$ is symmetric around zero for any $\gamma$, the condition $E=0$ implies that the longitudinal energies also come in opposite pairs and therefore the momenta in pairs $\left(\theta_{\mu}, \pi-\theta_{\mu}\right)$, which also violates the Assumption.

\footnotetext{
${ }^{4}$ As visible from the proofs, the convergence actually holds on pathspace equipped with the Skorohod topology. This obviously implies convergence of single-time distributions.
} 
Proposition 3 (Convergence to the DMPK process) Let $H_{\perp}$ be of the form (38) with $\gamma$ and $h_{\perp}$ such that Assumptions 1 and 2 hold. Let $\mathcal{A}(s)=\mathcal{A}(s)^{\gamma, h_{\perp}}$ be the process defined by Proposition 2. Choose a sequence $h_{\perp}(n)$ such that for any $n$, Assumptions 1 and 2 are satisfied and $h_{\perp}(n) \searrow 0$ as $n \nearrow \infty$. Then

$$
\mathcal{A}(s)^{\gamma, h_{\perp}(n)} \underset{\mathrm{distr}}{\stackrel{n \nearrow \infty}{\longrightarrow}} \mathcal{M}(s / c),
$$

where $\mathcal{M}(s)$ is the solution of the DMPK-SDE (15) and $c=c(E)$ is a constant that only depends on the energy $E$.

Note that the limit in (39) becomes independent of $\gamma$. Finally, by combining Propositions 2 and 3, one gets

$$
\lim _{h_{\perp}(n) \searrow 0 \lambda \searrow 0} \lim _{\lambda}\left(A^{\lambda}\left(\left\lfloor\lambda^{-2} s\right\rfloor\right)\right)=\mathcal{M}(s / c),
$$

namely the convergence of the microscopic Hamiltonian model to the DMPK random matrix model. The order of limits cannot be exchanged, since the bare limit $h_{\perp}(n) \searrow 0$ eliminates the transverse motion and hence all interaction between the channels. In fact, if $h_{\perp}=0$, then the model consists of $N$ copies of a one-dimensional chain and hence its localization length is independent of $N$. The rescaling constant $c=c(E)$ originates from the fact that our length unit $\lambda^{-2}$ is not precisely equal to the mean free path; see the footnote on Sect. 1.1.

\section{Discussion}

\subsection{Related Work and Outlook}

\subsubsection{Scaling Limits in the Anderson Model}

The existence of the metallic regime in the Anderson model in dimension $d>2$ is a major open problem of mathematical physics. An important step ahead was taken with the establishment of kinetic [10,25] and diffusive [12] scaling limits, where time and space are rescaled by appropriate powers of the coupling strengh $\lambda$, as the latter goes to 0 . In those scaling limits, the unitary evolution goes over to the linear Boltzmann equation or the diffusion equation. In spirit, our result is comparable to this approach, although the quasi one-dimensional metallic regime that we study is distinct from the one in $d>2$. In particular, the quasi 1D metallic regime exists in the scaling limit only (see the remarks following the Conjectures in Sect. 1.1). We have a kinetic space rescaling $x \mapsto \lambda^{-2} x$, in which the Hamiltonian model with disorder reduces to a fully stochastic model, just as the unitary evolution reduces to the fully stochastic Boltzmann equation in the works mentioned above. To make our results complete, we should establish that the stochastic models obtained in Propositions 2 and 3 do possess the properties of the metallic regime. This seems straightforward for the scaling limit (39), since the eigenvalue process associated with this SDE is the DMPK equation, which has been studied extensively in the physics literature, see Sect. 1. In fact, the random matrix ensemble that solves the SDE (15) is often mentioned on a par with the more familiar Dyson classes of random matrices, see e.g. [5]. To establish that the SDE obtained in Proposition 2 also has the universal properties of the metallic regime seems a more challenging task. In some sense, this is comparable to proving that e.g. Hermitian Wigner matrices with fully broken time-reversal invariance share the universality properties of GUE, as was recently established in $[11,13]$. 


\subsubsection{Alternative Models for the DMPK Theory}

Several models have been constructed to provide an alternative justification for the DMPK theory. Dorokhov [7] has constructed a Hamiltonian model of $N$ chains coupled only by random hopping acting strictly transversally. For each pair of chains $(\mu, v)$, the hopping potential $U_{\mu \nu}(x)$ at point $x$ is a Gaussian variable of zero mean and independent of all other pairs. The model is therefore invariant under the orthogonal group by construction. In fact, it is very similar to Wegner's $N$-orbitals model [29]. Another model that reproduces the DMPK theory is the (1D) supersymmetric nonlinear $\sigma$ model, [9].

\subsubsection{Lyapunov Exponents}

In [23], Schulz-Baldes calculated the lowest Lyapunov exponents for the Anderson model on a strip, at small disorder. As already remarked, this yields a partial confirmation of Conjecture 1 in Sect. 1.1. The drawback of this approach is that, by their very nature, Lyapunov exponents do not reveal much information on the metallic regime of disordered wires, although one could argue that the conjectured equidistancy of the Lyapunov spectrum is a sign of universality. In a recent work, the authors of [24] conjecture (and confirm numerically) that a certain property, the Random Phase Property (RPP), holds for the Anderson model on a strip. They remark that the RPP is weaker than the maximal entropy assumptions (see Sect. 1.3.2) made in the DMPK theory, yet it allows to estimate the Lyapunov exponents and hence the localization lengths. It is not clear to us (but it does not sound unreasonable) that the RPP in fact also implies universal behavior in the metallic regime. Further, it seems plausible that the transfer matrix ensembles that we obtain in Proposition 2 satisfy the RPP.

\subsubsection{Hyperbolic Brownian Motion}

The DMPK equation (24) for $N=1$ was solved explicitly in [16]. It can be related to Brownian motion in the hyperbolic plane. A very similar description can be given from the point of view of the Brownian Carousel [27]. As already mentioned, the DMPK equation for any $N$ can be related to Brownian motion on symmetric spaces, see [18, 30].

\subsection{Idea of the Scaling Limit}

We now explain heuristically why the propositions hold true. Recall the definition of $A^{\lambda}(x), x \in \mathbb{N}$ in (32), i.e. $A^{\lambda}(x):=\left[M^{0}\right]^{-x} M^{\lambda}(x)$. For convenience, we define $G$ such that

$$
M^{0}=\exp (\mathrm{i} G), \quad \text { hence } G=\left(\begin{array}{cc}
\theta\left(E_{\|}\right) & 0 \\
0 & -\theta\left(E_{\|}\right)
\end{array}\right),
$$

and we drop the $\lambda$-dependence on $A^{\lambda}(x)$. Then, $A(x)$ satisfies the stochastic difference equation

$$
A(x+1)-A(x)=\underbrace{\left[\mathrm{e}^{-\mathrm{i}(x+1) G} \Upsilon T_{x+1}^{\lambda} \Upsilon^{-1} \mathrm{e}^{\mathrm{i} x G}-1\right]}_{:=\lambda Z_{x+1}} A(x) .
$$

We remark that the matrices $Z_{x+1}$ are independent of $A(x)$ and $\mathbb{E}\left(Z_{x+1}\right)=0$, hence $A(x)$ is a discrete-time martingale. The $Z_{x}$ are however oscillating in $x$ due to the factors $\mathrm{e}^{\mathrm{i} x G}$. We 
can write

$$
Z_{x}=e^{-\mathrm{i} x G} R_{x} e^{\mathrm{i} x G}, \quad \lambda R_{x}:=e^{-\mathrm{i} G} \Upsilon T_{x}^{\lambda} \Upsilon^{-1}-1,
$$

where $R_{x}$ are i.i.d. matrix-valued random variables. From the explicit formula for $T_{x}^{\lambda}$, we can now check that the expression is indeed linear in $\lambda$. Note also that the $2 N \times 2 N$ matrix $Z_{x}$ contains only $N$ independent random variables, namely the $v_{x, z}, z=1, \ldots, N$.

To gain some insight, we first replace $Z_{x}$ by $R_{x}$. That is, we consider the process $D(x)$ defined by

$$
D(x+1)-D(x)=\lambda R_{x+1} D(x), \quad D(0)=1 .
$$

First we remark that, with appropriate conditions on the random variable $v_{x, z}$, we have

$$
\lambda \sum_{x=0}^{\left\lfloor\lambda^{-2} s\right\rfloor} R_{x} \underset{\operatorname{distr}}{\stackrel{\lambda \searrow 0}{\longrightarrow}} \mathcal{R}(s), \quad \text { for all } s
$$

where the matrix-valued Brownian motion $\mathcal{R}(s)$ is defined by replacing each random variable $v_{x, z}$ in the definition of $R_{x}$ by the Brownian motion $B_{z}(s)$. Here $z$ is the index ranging from 1 to $N$. Of course, (42) is nothing more than a multidimensional version of the convergence of random walk to Brownian motion. We emphasize that the $2 N \times 2 N$ matrix entries of $\mathcal{R}(x)$ are a linear combination of $N$ independent Brownian motions only. It is not hard to believe that the discrete-time process (41) converges to the solution of the corresponding SDE

$$
D\left(\left\lfloor\lambda^{-2} s\right\rfloor\right) \underset{\operatorname{distr}}{\stackrel{\lambda \searrow 0}{\longrightarrow}} \mathcal{D}(s), \quad \text { where } d \mathcal{D}(s)=d \mathcal{R}(s) \mathcal{D}(s), \mathcal{D}(0)=1 .
$$

as is easily proven by standard martingale theory, see e.g. [14] and Sect. 4.

We now look for the analogue of the convergence (42) upon replacing $R_{x}$ by $Z_{x}$. Let us first observe it, for example, at the level of the second moment. Indeed,

$$
\mathbb{E}\left[\left(\lambda \sum_{x=1}^{\left\lfloor\lambda^{-2} s\right\rfloor} Z_{x}\right)_{i j}\left(\lambda \sum_{x^{\prime}=1}^{\left\lfloor\lambda^{-2} s\right\rfloor} Z_{x^{\prime}}\right)_{k l}\right]=\lambda^{2} \sum_{x=1}^{\left\lfloor\lambda^{-2} s\right\rfloor} \mathrm{e}^{-\mathrm{i} x\left(G_{i}-G_{j}+G_{k}-G_{l}\right)} \mathbb{E}\left[\left(R_{x}\right)_{i j}\left(R_{x}\right)_{k l}\right]
$$

where the indices $i, j, k, l$ refer to a basis in which the matrix $G$ is diagonal and the numbers $G_{i}, G_{j}, \ldots$ are the eigenvalues. This is hence the basis in which the free transfer matrix is diagonal, and the base vectors can be indexed by the double index $(\mu, q)$ where $\mu \in$ $\{1, \ldots, N\}$ indexes the channels and $q \in\{+1,-1\}$ is +1 for the left moving and -1 for the right moving sectors. The eigenvalues are $G_{j}=q \theta_{\mu}$ if $j=(\mu, q)$. Since $\mathbb{E}\left[\left(R_{x}\right)_{i j}\left(R_{x}\right)_{k l}\right]$ is independent of $x,(43)$ converges to

$$
s \cdot \delta_{G_{i}-G_{j}, G_{l}-G_{k}} \mathbb{E}\left[(R .)_{i j}(R .)_{k l}\right] .
$$

Thus, one can check that

$$
\lambda \sum_{x=1}^{\left\lfloor\lambda^{-2} s\right\rfloor} Z_{x} \underset{\operatorname{distr}}{\stackrel{\lambda \searrow 0}{\longrightarrow}} \mathcal{Z}(s), \quad \text { for all } s,
$$


where $\mathcal{Z}(s)$ is a matrix valued $\mathrm{BM}$ characterized by the covariances

$$
\begin{aligned}
& \mathbb{E}\left(\mathcal{Z}_{i j}(s) \mathcal{Z}_{k l}(s)\right)=\delta_{G_{i}-G_{j}, G_{l}-G_{k}} \mathbb{E}\left(\mathcal{R}_{i j}(s) \mathcal{R}_{k l}(s)\right), \\
& \mathbb{E}\left(\mathcal{Z}_{i j}^{*}(s) \mathcal{Z}_{k l}(s)\right)=\delta_{G_{i}-G_{j}, G_{l}-G_{k}} \mathbb{E}\left(\mathcal{R}_{i j}^{*}(s) \mathcal{R}_{k l}(s)\right) .
\end{aligned}
$$

It is in this place that we need to use Assumption 2. Indeed, Assumption 2 states that the eigenvalues of the matrix $G$ do not have any type of degeneracy, hence a condition like $\left(G_{i}-G_{j}+G_{k}-G_{l}\right)=0$ is only satisfied when $i=k, j=l$ or $i=j, k=l$. In other words, the fast oscillations originating from the free part of the transfer matrix kill most of the correlations between matrix elements of $\mathcal{Z}(s)$. As a result, the number of independent random variables in $\mathcal{Z}(s)$ is $O\left(N^{2}\right)$ whereas in $\mathcal{R}(s)$ it was $O(N)$. In [28], this phenomenon is called 'noise explosion'.

Next, we calculate the nonvanishing covariances of $\mathcal{Z}(s)$ arising from our microscopic model. The matrix $R_{x}$ is given explicitly by

$$
\begin{aligned}
\lambda R_{x} & =\mathrm{e}^{-\mathrm{i} G} \Upsilon\left(O^{*} \otimes 1_{2}\right)\left(\begin{array}{cc}
E-H_{x x} & -1_{N} \\
1_{N} & 0
\end{array}\right)\left(O \otimes 1_{2}\right) \Upsilon^{-1}-M_{x}^{0} \\
& =-\lambda \mathrm{e}^{-\mathrm{i} G} \Upsilon\left(\begin{array}{cc}
\widetilde{V}_{x} & 0 \\
0 & 0
\end{array}\right) \Upsilon^{-1}
\end{aligned}
$$

where $\widetilde{V}_{x}=\widetilde{V}_{x}^{*}$ was defined in Proposition 2. Hence

$$
\mathbb{E}\left(\left(R_{x}^{*}\right)_{j i}\left(R_{x}\right)_{i j}\right)=\mathbb{E}\left|\left(\Upsilon\left(\begin{array}{cc}
\tilde{V}_{x} & 0 \\
0 & 0
\end{array}\right) \Upsilon^{-1}\right)_{i j}\right| .
$$

Recalling the definition of the map $\Upsilon$, we can write it explicitly as a matrix with diagonal $N \times N$ blocks.

$$
\Upsilon=\left(\begin{array}{c|c}
\left(\mathrm{i} \rho\left(E_{\|}\right)\right)^{-1 / 2} & \left(\mathrm{i} \rho\left(E_{\|}\right)\right)^{-1 / 2} \\
\hline\left(\mathrm{i} \rho\left(E_{\|}\right)\right)^{-1 / 2} \exp \left(-\mathrm{i} \theta\left(E_{\|}\right)\right) & \left(\mathrm{i} \rho\left(E_{\|}\right)\right)^{-1 / 2} \exp \left(\mathrm{i} \theta\left(E_{\|}\right)\right)
\end{array}\right),
$$

where $\rho\left(E_{\|}\right)=2 \sin \theta\left(E_{\|}\right)$. By direct calculation, we can now check that the covariance of $\mathcal{Z}(s)$ agrees with that of the RHS of (37), i.e.

$$
\mathbb{E}\left|\mathcal{Z}_{i j}\right|^{2}=\mathbb{E}\left|\left(R_{x}\right)_{i j}\right|^{2}=\left|\sigma_{\mu \nu}\right|^{2}, \quad i=(\mu, \pm), j=(\nu, \pm) .
$$

We now explain heuristically how this gives rise to Proposition 2. Recall that $A(\cdot)$ satisfies the stochastic difference equation

$$
A(x+1)-A(x)=\lambda Z_{x+1} A(x) .
$$

Since $Z_{x}$ has zero mean and unit variance, it takes a time of order $\lambda^{-2}$ for $A(\cdot)$ to change appreciably. However, on this timescale, the oscillations in $Z$. are not longer visible and one can hence replace $\lambda Z_{\lfloor\lambda-2 s\rfloor} \approx d \mathcal{Z}(s)$, i.e., by a time average on the fast time-scale, in (46). This suggests that the scaling limit $\mathcal{A}_{s}$ of $A\left(\left\lfloor\lambda^{-2} s\right\rfloor\right)$, if it exists, must be a solution of $d \mathcal{A}(s)=d \mathcal{Z}(s) \mathcal{A}(s)$, and hence Proposition 2 holds. 


\section{Proof of the Scaling Limits}

Proposition 2 is essentially a textbook result in homogenization theory, where one starts from an evolution equation with a fast degree of freedom that evolves independently of the slow degrees. In our case, the fast degrees of freedom are the oscillating phases in the random variables $Z_{x}$. For a treatment of these matters, we refer the reader to [15], where one discusses a setup identical to our case (see Chaps. 6 and 7 of [15]). In fact, the only difference is that our model is defined on the lattice instead of in the continuum (and hence we can have independent random potentials instead of a rapidly decaying dependence in the $x$ variable). One can mimick the proof of [15] apart from the fact that we have to establish tightness of processes with sample paths in $D_{\mathbb{R}^{d}}\left[0, \infty\left[\right.\right.$ instead of $C_{\mathbb{R}^{d}}[0, \infty[$.

For the sake of explicitness, we outline a proof based on an expansion. We first show that the second, third and fourth moments of $A_{x}$ converge to those of $\mathcal{A}(s)$ in a scaling limit. Then, in Sect. 4.2, we invoke a standard result to eventually prove in Sect. 4.3 that the convergence of moments is sufficient to conclude the proof.

\subsection{Convergence of Moments}

We will establish the convergence of fourth moments of $A^{\lambda}\left(\left\lfloor\lambda^{-2} s\right\rfloor\right)$ by a brutal, but completely standard estimate. We use the assumption that the third and fourth moments $\mathbb{E}\left(v^{3}\right), \mathbb{E}\left(v^{4}\right)$ are finite.

Lemma 4 Let $P_{e}, e=a, b, c, d$ be operators on $\mathbb{C}^{2 N}$ with $\left\|P_{e}\right\|=1$ and let $s_{e} \in[0, \tau]$, $e=a, b, c, d$ for some finite $\tau$.

Let $\left(A^{\lambda}(x)\right)^{\#}$ stand for either $\left(A^{\lambda}(x)\right)^{*}$ or $A^{\lambda}(x)$. Then

$$
\begin{aligned}
& \mid \mathbb{E}\left[\operatorname{Tr} P_{a}\left(A^{\lambda}\left(\left\lfloor\lambda^{-2} s_{a}\right\rfloor\right)\right)^{\#} P_{b}\left(A^{\lambda}\left(\left\lfloor\lambda^{-2} s_{b}\right\rfloor\right)\right)^{\#} P_{c}\left(A^{\lambda}\left(\left\lfloor\lambda^{-2} s_{c}\right\rfloor\right)\right)^{\#} P_{d}\left(A^{\lambda}\left(\left\lfloor\lambda^{-2} s_{d}\right\rfloor\right)\right)^{\#}\right] \\
& \quad-\mathbb{E}\left[\operatorname{Tr} P_{a}\left(\mathcal{A}\left(s_{a}\right)\right)^{\#} P_{b}\left(\mathcal{A}\left(s_{b}\right)\right)^{\#} P_{c}\left(\mathcal{A}\left(s_{c}\right)\right)^{\#} P_{d}\left(\mathcal{A}\left(s_{d}\right)\right)^{\#}\right] \mid \leq C(\lambda, \tau, N)
\end{aligned}
$$

where $C(\lambda, \tau, N) \searrow 0$ as $\lambda \searrow 0$.

Proof To avoid too many constants, we will treat the case where $s_{e}=\tau, P_{e}=1$ and \# are chosen such that we take the adjoint of the first and third $A$. The general case is completely analogous.

By iterating the difference equation (40), we obtain

$$
(47)=\mathbb{E}\left(\operatorname{Tr} \prod_{x_{a}=1}^{\tau_{\lambda}}\left(1+\lambda Z_{x_{a}}^{*}\right) \prod_{x_{b}=1}^{\tau_{\lambda}}\left(1+\lambda Z_{x_{b}}\right) \prod_{x_{c}=1}^{\tau_{\lambda}}\left(1+\lambda Z_{x_{c}}^{*}\right) \prod_{x_{d}=1}^{\tau_{\lambda}}\left(1+\lambda Z_{x_{d}}^{*}\right)\right)
$$

where we have abbreviated $\tau_{\lambda}:=\left\lfloor\lambda^{-2} \tau\right\rfloor$ and the $Z_{x_{e}}$ are ordered with decreasing indices and the $Z_{x_{e}}^{*}$ have increasing indices. Evaluating the products yields

$$
(49)=1+\sum_{n>0} \lambda^{n} \sum_{P_{n}} \underbrace{\mathbb{E} \operatorname{Tr}\left(\left(Z_{x_{a}^{1}}^{*} \cdots Z_{x_{a}^{m_{a}}}^{*}\right)\left(Z_{x_{b} m_{b}} \cdots Z_{x_{b}^{1}}\right)\left(Z_{x_{c}^{1}}^{*} \cdots Z_{x_{c}^{m_{c}}}^{*}\right)\left(Z_{x_{d}^{m_{d}}} \cdots Z_{x_{d}^{1}}\right)\right)}_{r\left(P_{n}\right)} \text {. }
$$

In (50), $1 \leq x_{e}^{1}<\cdots<x_{e}^{m_{e}} \leq \tau_{\lambda}$ are sites (elements of $\mathbb{N}$ ), for $e=a, b, c, d$ and some numbers $m_{a}+m_{b}+m_{c}+m_{d}=n$. The sum $\sum_{P_{n}}$ runs over all such sets of sites, such that the $\mathbb{E}(\cdot)$ is not zero, i.e. such that for each $x_{e}^{i}$ that appears in the set, there is at least one 
partner $x_{e^{\prime}}^{i}=x_{e}^{i}$ with $e^{\prime} \neq e$. This allows us to divide the sites that appear in the sets into three classes: those that appear twice, three or four times. Let $n_{2}, n_{3}, n_{4}$ be the number of such sites. Then $2 n_{2}+3 n_{3}+4 n_{4}=n$. The number of $P_{n}$ corresponding to $\left(n_{2}, n_{3}, n_{4}\right)$ is estimated as

$$
\#\left\{P_{n}:\left(n_{2}, n_{3}, n_{4}\right) \text { is fixed }\right\} \leq \frac{\left(\tau_{\lambda} C_{2}^{4}\right)^{n_{2}}}{n_{2} !} \cdot \frac{\left(\tau_{\lambda} C_{3}^{4}\right)^{n_{3}}}{n_{3} !} \cdot \frac{\left(\tau_{\lambda} C_{4}^{4}\right)^{n_{4}}}{n_{4} !},
$$

where $C_{2,3,4}^{4}$ are binomial coefficients. For each choice of $P_{n}$,

$$
\left|r\left(P_{n}\right)\right| \leq\left(N \mathbb{E}\left(v^{2}\right)\right)^{n_{2}} \cdot\left(N \mathbb{E}\left(v^{3}\right)\right)^{n_{3}} \cdot\left(N \mathbb{E}\left(v^{4}\right)\right)^{n_{4}} .
$$

This gives us an a-priori bound on the sum in (50):

$$
\begin{aligned}
\sum_{n} & \lambda^{n} \sum_{P_{n}}\left|r\left(P_{n}\right)\right| \\
\leq & \sum_{n_{2}, n_{3}, n_{4} \geq 0} \lambda^{2 n_{2}+3 n_{3}+4 n_{4}} \sum_{P_{n}:\left(n_{2}, n_{3}, n_{4}\right) \text { fixed }}\left|r\left(P_{n}\right)\right| \\
\leq & \sum_{n_{2}, n_{3}, n_{4} \geq 0} \lambda^{2 n_{2}+3 n_{3}+4 n_{4}} \cdot \frac{\left(C_{2}^{4} \mathbb{E}\left(v^{2}\right) N \tau_{\lambda}\right)^{n_{2}}}{n_{2} !} \cdot \frac{\left(C_{3}^{4} \mathbb{E}\left(v^{3}\right) N \tau_{\lambda}\right)^{n_{3}}}{n_{3} !} \cdot \frac{\left(C_{4}^{4} \mathbb{E}\left(v^{4}\right) N \tau_{\lambda}\right)^{n_{4}}}{n_{4} !} \\
\leq & \sum_{n_{2}, n_{3}, n_{4} \geq 0} \frac{(\text { const } \cdot N \tau)^{n_{2}}}{n_{2} !} \cdot \frac{(\text { const } \cdot \lambda N \tau)^{n_{3}}}{n_{3} !} \cdot \frac{\left(\text { const } \cdot \lambda^{2} N \tau\right)^{n_{4}}}{n_{4} !} \\
\leq & \mathrm{e}^{\text {const } \cdot N \tau}, \quad \text { for any } \lambda .
\end{aligned}
$$

Hence, by dominated convergence, we can interchange the sum over $n$ and the limit $\lambda \searrow 0$ to obtain

$$
\text { (49) }=1+\sum_{n>0} \lim _{\lambda \searrow 0} \lambda^{n} \sum_{P_{n}} r\left(P_{n}\right) .
$$

One can also see immediately that all terms with $n_{3} \neq 0$ or $n_{4} \neq 0$ vanish as $\lambda \searrow 0$. Therefore, the sum over $P_{n}$ reduces to a sum over pairings, each involving $n=2 v$ matrices $Z_{x}^{m}$, and the expectation value is a simple product of $v$ covariances. Each such pairing can be described by first specifying a 'pattern', namely from which group $a, b, c, d$ the matrices are drawn in each pair, and then the particular point $x$ associated to each pair. Given a pattern, we can use the same oscillatory argument that led to (44) to handle the limit of the sum over all sets of points satisfying that pattern, thereby replacing sums over $Z_{x_{e}}^{m_{e}}$ by stochastic integrals over Brownian motions $d \mathcal{Z}\left(s_{e}^{m_{e}}\right)$ (recall that the Kronecker $\delta$ 's are included in the definition of $\mathcal{Z}(s)$ ). Hence,

$$
\begin{aligned}
\lim _{\lambda \searrow 0} \lambda^{2 v} & \sum_{P_{2 v}} r\left(P_{2 v}\right) \\
= & \sum_{m_{a}+m_{b}+m_{c}+m_{d}=2 v} \mathbb{E}\left(\operatorname{Tr} \int_{0<s_{a}^{1}<\cdots<s_{a}^{m_{a}<\tau}} d \mathcal{Z}^{*}\left(s_{a}^{1}\right) \ldots d \mathcal{Z}^{*}\left(s_{a}^{m_{a}}\right)\right. \\
& \times \int_{0<s_{b}^{1<\cdots<s_{b}^{m}<\tau}} d \mathcal{Z}\left(s_{b}^{m_{b}}\right) \ldots d \mathcal{Z}\left(s_{b}^{1}\right) \cdot \int_{0<s_{c}^{1}<\cdots<s_{c}^{m_{c}<\tau}} d \mathcal{Z}^{*}\left(s_{c}^{1}\right) \ldots d \mathcal{Z}^{*}\left(s_{c}^{m_{c}}\right) \\
& \left.\times \int_{0<s_{d}^{1}<\cdots<s_{d}^{m_{d}}<\tau} d \mathcal{Z}\left(s_{d}^{m_{d}}\right) \ldots d \mathcal{Z}\left(s_{d}^{1}\right)\right) .
\end{aligned}
$$


As an illustrative example of the discussion above, we perform the computation explicitly in the case $m_{a}=m_{b}=2, m_{c}=m_{d}=0$. Then

$$
\begin{aligned}
\lambda^{2} r\left(P_{2+2+0+0}\right) & =\lambda^{2} \sum_{x_{a}^{1,2}, x_{b}^{1,2}} \mathbb{E}\left(\operatorname{Tr}\left(Z_{x_{a}^{1}}^{*} Z_{x_{a}^{2}}^{*} \cdot Z_{x_{b}^{2}} Z_{x_{b}^{1}}\right)\right) \\
& =\lambda^{2} \sum_{1 \leq x^{1}<x^{2} \leq \tau_{\lambda}} \mathbb{E}\left(\operatorname{Tr}\left(Z_{x^{1}}^{*} Z_{x^{2}}^{*} Z_{x^{2}} Z_{x^{1}}\right)\right) \\
& \stackrel{\lambda \unlhd 0}{\longrightarrow} \mathbb{E}\left(\operatorname{Tr} \int_{0 \leq s^{1}<s^{2} \leq \tau} d \mathcal{Z}\left(s^{1}\right)^{*} d \mathcal{Z}\left(s^{2}\right)^{*} d \mathcal{Z}\left(s^{2}\right) d \mathcal{Z}\left(s^{1}\right)\right) \\
& =\mathbb{E}\left(\operatorname{Tr} \int_{0}^{\tau} \int_{0}^{s_{a}^{2}} d \mathcal{Z}\left(s_{a}^{1}\right)^{*} d \mathcal{Z}\left(s_{a}^{2}\right)^{*} \cdot \int_{0}^{\tau} \int_{0}^{s_{b}^{2}} d \mathcal{Z}\left(s_{b}^{2}\right) d \mathcal{Z}\left(s_{b}^{1}\right)\right)
\end{aligned}
$$

where (51) follows from the convergence of the second moments, (44), and the Gaussian property of the Brownian increments, and (52) by their independence for unequal times. We note that the contraction pattern

$$
Z_{x_{a}^{1}}^{*} Z_{x_{a}^{2}}^{*} Z_{x_{b}^{2}} Z_{x_{b}^{1}}
$$

does not appear because of the ordering of the matrix product. Moreover, the iterated Itointegrals are well-defined since $\int_{0}^{s_{a}^{2}} d \mathcal{Z}\left(s_{a}^{1}\right)$ is an Ito-process which is adapted to the filtration associated with the Brownian motion $\mathcal{Z}\left(s_{a}^{2}\right)$.

To conclude, we observe that the summation over $n$ yields the stochastic Neumann series associated to the SDE (34) in each of the four brackets, and therefore (48).

In fact, one could repeat this proof for all moments if one assumes that all moments of $v_{x, z}$ exist.

\subsection{An Abstract Result on Convergence to Diffusions}

Let $X^{n}(t), n \in \mathbb{N}$, be a sequence of $\mathbb{R}^{d}$-valued discrete time martingales, defined via the discrete time stochastic difference equation

$$
X^{n}\left(t+\lambda_{n}^{2}\right)-X^{n}(t)=\lambda_{n} \cdot \xi^{n}(t) X^{n}(t), \quad t \in \lambda_{n}^{2} \mathbb{N}
$$

where $\lambda_{n} \searrow 0$ as $n \nearrow \infty$, and $\xi^{n}(t)=\left(\xi_{i j}^{n}(t)\right)_{i, j=1}^{d}$ are random variables with mean zero and covariance

$$
\mathbb{E}\left(\xi_{i j}^{n}(t) \xi_{k l}^{n}\left(t^{\prime}\right)\right)=\delta_{t, t^{\prime}} C_{i, j, k, l}^{n}(t), \quad t, t^{\prime} \in \lambda_{n}^{2} \mathbb{N} .
$$

We assume that the functions $C_{i, j, k, l}^{n}(\cdot)$ are uniformly bounded and

$$
\int_{0}^{\tau} d s f(s) C_{i, j, k, l}^{n}(s) \stackrel{n \nearrow \infty}{\longrightarrow} \sigma_{i j} \sigma_{k l} \int_{0}^{\tau} d s f(s), \quad \text { for any } f \in \mathcal{C}(\mathbb{R}) .
$$

The jump process $X_{n}$ is viewed as a process in continuous time whose sample paths belong to $D_{\mathbb{R}^{d}}[0, \infty$ [, the space of right-continuous functions with left limits, equipped with the Skorohod topology. The set of jump times $t_{J}$ is thus $\lambda_{n}^{2} \mathbb{N}$. In this context, we shall denote by $X^{n}\left(t_{J}-\right)$ the left limit of the sample path of $X^{n}$ at $t_{J}$. 
The following lemma is a version of Theorem 4.1 in Chap. 7 of [14], simplified such as to match the setup above.

Lemma 5 (Convergence to diffusions) Let $\mathcal{X}$ be a $\mathbb{R}^{d}$-valued diffusion process $\mathcal{X}$ defined as the unique strong solution of the SDE

$$
d \mathcal{X}_{i}(s)=\sum_{j} \sigma_{i j} \mathcal{X}_{j}(s) d B_{j}(s), \quad \mathcal{X}(0)=c \in \mathbb{R}^{d},
$$

with respect to the filtration generated by the d-dimensional Brownian motion $B(s)$. Assume that

- The processes

$$
W_{i j}^{n}(s):=\sum_{k, l} C_{i, k, j, l}^{n}(s) X_{k}^{n}(s) X_{l}^{n}(s)
$$

satisfy

$$
\sup _{0 \leq t \leq \tau} \mid \underbrace{\int_{0}^{t} d s\left(W_{i j}^{n}(s)-\sum_{k, l} \sigma_{i k} \sigma_{j l} X_{k}^{n}(s) X_{l}^{n}(s)\right) \mid}_{=: Y_{i j}^{n}(t)} \underset{\text { distr }}{\stackrel{n \nearrow \infty}{\longrightarrow}} 0 ;
$$

- The jumps of $X^{n}$ become small in the sense that

$$
\begin{aligned}
& \mathbb{E}\left(\sup _{t_{J} \in[0, \tau]}\left|X_{i}^{n}\left(t_{J}\right)-X_{i}^{n}\left(t_{J}-\right)\right|^{2}\right) \longrightarrow 0, \\
& \mathbb{E}\left(\sup _{t_{J} \in[0, \tau]}\left|W_{i j}^{n}\left(t_{J}\right)-W_{i j}^{n}\left(t_{J}-\right)\right|\right) \longrightarrow 0, \quad n \nearrow \infty,
\end{aligned}
$$

where the sup ranges over all jump time $t_{J}$.

Then,

$$
X^{n} \underset{\text { distr }}{\stackrel{n \nearrow \infty}{\longrightarrow}} \mathcal{X}
$$

on the space $D_{\mathbb{R}^{d}}[0, \infty[$.

Note that

$$
X_{i}^{n}(t) X_{j}^{n}(t)-\int_{0}^{t} d s W_{i j}^{n}(s)
$$

are martingales with respect to the filtration generated by $X^{n}(t)$, as required by Theorem 4.1 in Chap. 7 of [14].

\subsection{Proof of Proposition 2}

To prove Proposition 2, we use Lemma 5 with $X^{n}(s)$, resp. $\mathcal{X}(s)$, the $\mathbb{R}^{d}$-valued processes containing all real and imaginary parts of the matrix elements of $A^{\lambda}\left(\left\lfloor\lambda^{-2} s\right\rfloor\right)$, resp. $\mathcal{A}(s)$, (hence $d=2(2 N)^{2}$ ) and with $n$ labeling a sequence $\lambda_{n}$ such that $\lambda_{n} \searrow 0$ as $n \nearrow \infty$. The functions $C_{i, j, k, l}^{n}(\cdot)$ are the oscillating covariances of the random variables $Z_{x}$, see Sect. 3.2.

Lemma 4 yields that all 4 th moments of $X^{n}$ converge to those of $\mathcal{X}$ as $n \nearrow \infty$, uniformly on compacts, i.e.

$$
\sup _{\left\{s_{i}\right\} \in\left[\tau, \tau^{\prime}\right]}\left[\mathbb{E}\left(X_{i_{1}}^{n}\left(s_{1}\right) X_{i_{2}}^{n}\left(s_{2}\right) X_{i_{3}}^{n}\left(s_{3}\right) X_{i_{4}}^{n}\left(s_{4}\right)\right)-\mathbb{E}\left(\mathcal{X}_{i_{1}}\left(s_{1}\right) \mathcal{X}_{i_{2}}\left(s_{2}\right) \mathcal{X}_{i_{3}}\left(s_{3}\right) \mathcal{X}_{i_{4}}\left(s_{4}\right)\right)\right] \longrightarrow 0,
$$


and we note that the same argument can be repeated to provide the convergence of 2 nd and 3rd moments.

We check condition (54) of Lemma 5. First, by the definition of $Y^{n}$, and using the uniform bound on the fourth moments of $X^{n}$, we observe that for any $t, t^{\prime} \in[0, \tau]$ and for all $i, j=$ $1, \ldots, d$,

$$
\mathbb{E}\left|Y_{i j}^{n}\left(t^{\prime}\right)-Y_{i j}^{n}(t)\right|^{2} \leq\left(t-t^{\prime}\right)^{2} C,
$$

and $C<\infty$ can be chosen independent of $n$. The Chebyshev inequality then reads

$$
\mathbb{P}\left(\left|Y_{i j}^{n}\left(t^{\prime}\right)-Y_{i j}^{n}(t)\right|^{2}>b\right) \leq \frac{1}{b}\left(t-t^{\prime}\right)^{2} C .
$$

To show that this yields the convergence (54), let us assume that the random variable there does not vanish in distribution. That implies in particular that there is a sequence of times $t_{m} \in[0, \tau]$, and some constants $c, c^{\prime}>0$ such that;

$$
\mathbb{P}\left(\left|Y_{i j}^{m}\left(t_{m}\right)\right|>c\right)>c^{\prime}, \quad \text { for all } m .
$$

Let $\bar{t}$ be an accumulation point of the sequence $\left(t_{m}\right)$. Then (59) implies that either $\mathbb{P}\left(\left|Y_{i j}^{m}\left(t_{m}\right)-Y_{i j}^{m}(\bar{t})\right|>c / 2\right)>c^{\prime} / 2$, which is excluded by (58), or $\mathbb{P}\left(\left|Y_{i j}^{m}(\bar{t})\right|>c / 2\right)>c^{\prime} / 2$. But the latter can also be excluded by observing that, as $m \rightarrow \infty$,

$$
\mathbb{E}\left(\left|Y_{i j}^{m}(\bar{t})\right|^{2}\right) \longrightarrow 0 \text {. }
$$

This follows by the convergence of fourth moments, the continuity of sample paths of $\mathcal{X}$ and the weak convergence (53). Hence, condition (54) is proven.

We now turn to condition (55). Recalling that $X^{n}\left(t_{J}\right)-X^{n}\left(t_{J}-\right)=\lambda_{n} \cdot \xi^{n}\left(t_{J}\right) X^{n}\left(t_{J}\right)$ for any jump time $t_{J}$, we have for any $\alpha>0$ and $b>0$,

$$
\begin{aligned}
& \mathbb{P}\left(\sup _{t_{J} \in[0, \tau]}\left|X_{i}^{n}\left(t_{J}\right)-X_{i}^{n}\left(t_{J}-\right)\right|>b\right) \\
& \quad \leq \lambda_{n}^{-2} \cdot \sup _{t_{J}} \mathbb{P}\left(\left|\lambda_{n} \cdot \xi_{i j}^{n}\left(t_{J}\right) X_{j}^{n}\left(t_{J}\right)\right|>b\right) \\
& \quad \leq \lambda_{n}^{-2} \cdot \sup _{t_{J}} \frac{1}{b^{\alpha}} \mathbb{E}\left|\lambda_{n} \cdot \xi_{i j}^{n}\left(t_{J}\right) X_{j}^{n}\left(t_{J}\right)\right|^{\alpha} \\
& \quad \leq \frac{1}{b^{\alpha}} \cdot \lambda_{n}^{-2+\alpha} \cdot \sup _{t_{J}}\left(\mathbb{E}\left|\xi_{i j}^{n}\left(t_{J}\right)\right|^{\alpha a}\right)^{1 / a}\left(\mathbb{E}\left|X_{j}^{n}\left(t_{J}\right)\right|^{\alpha a^{\prime}}\right)^{1 / a^{\prime}}, \quad 1 / a+1 / a^{\prime}=1
\end{aligned}
$$

where the first equality follows from the fact that there are $\lambda_{n}^{-2}$ jumps in the interval $[0, \tau]$, the second and third are Chebyshev and Cauchy-Schwartz inequalities, respectively. If $a, a^{\prime}$ are chosen so that $\alpha a^{\prime} \leq 4$ and $\mathbb{E}\left|\xi_{i j}^{n}\left(t_{J}\right)\right|^{\alpha a}<\infty$, the last expression is bounded by

$$
\frac{1}{b^{\alpha}} \cdot O\left(\lambda_{n}^{-2+\alpha}\right)
$$

Let now $b>1$. To conclude, we compute

$$
\begin{aligned}
& \mathbb{E}\left(\sup _{t_{J} \in[0, \tau]}\left|X_{i}^{n}\left(t_{J}\right)-X_{i}^{n}\left(t_{J}-\right)\right|^{2}\right) \\
& \quad \leq \lambda_{n}^{\frac{-2+\alpha}{2 \alpha}}+\mathbb{P}\left(\left|H^{n}\right| \in\left[\lambda_{n}^{\frac{-2+\alpha}{2 \alpha}}, 1\right]\right)+\sum_{k=0}^{\infty} \mathbb{E}\left(\left|H^{n}\right|^{2}|| H^{n} \mid \in\left[b^{k}, b^{k+1}\right]\right) \cdot \mathbb{P}\left(\left|H^{n}\right| \in\left[b^{k}, b^{k+1}\right]\right) \\
& \quad \leq \lambda_{n}^{\frac{-2+\alpha}{2 \alpha}}+O\left(\lambda_{n}^{\frac{-2+\alpha}{2}}\right)+O\left(\lambda_{n}^{-2+\alpha}\right) \cdot \sum_{k=0}^{\infty} b^{2(k+1)} \cdot b^{-k \alpha}
\end{aligned}
$$


where we have abbreviated $H^{n}=\sup _{t_{J} \in[0, \tau]}\left|X_{i}^{n}\left(t_{J}\right)-X_{i}^{n}\left(t_{J}-\right)\right|$. Condition (55) follows by noting that the choice $\alpha>2$ ensures both the finiteness of the series and the convergence of the expectation value to zero. Finally, Condition (56) follows by a similar argument. This concludes the proof of Proposition 2.

Note that $\alpha>2$ implies $a>2$ and therefore $\alpha a>4$, whence the need to control higher moments of the potential $v$, see (26).

\subsection{Proof of Proposition 3}

To prove Proposition 3, we note that, for $H_{\perp}$ as in (38), the basis of eigenfunctions $\psi_{\mu}$ consists simply of the exponential functions on $\mathbb{Z} / N \mathbb{Z}$, independently of $\gamma$. Hence,

$$
\mathbb{E}\left|\tilde{V}_{\mu \nu}\right|^{2}=1 / N, \quad \text { independently of } \mu, \nu .
$$

Since $h_{\perp} \searrow 0$, also $E_{\perp}(\mu) \searrow 0$ for all $\mu$ and hence $E_{\|}(\mu) \rightarrow E$ and $\theta_{\mu} \rightarrow \theta$ where $\theta$ is the solution of $2 \cos \theta=E$. This means that

$$
\left(\sigma_{\mu \nu}\right)^{2} \rightarrow \frac{1}{4\left(1-(\cos \theta)^{2}\right)}=\frac{1}{4\left(1-(E / 2)^{2}\right)},
$$

which fixes the scale factor $c$ in the RHS of (39). Since both the processes $\mathcal{A}$ and $\mathcal{M}$ are solutions of SDE's with smooth and uniformly bounded coefficients, convergence of the solutions follows from convergence of the coefficients, see e.g. Chap. 11 in [26].

Acknowledgements This work was started while both authors were in the group of G.M. Graf at the ETH Zürich. We enthusiastically thank Gian Michele for many insights and discussions on the topic of this paper. S.B. is supported by the National Science Foundation under Grant DMS-0757581 and W.D.R is partially supported by the European Research Council and the Academy of Finland.

Open Access This article is distributed under the terms of the Creative Commons Attribution Noncommercial License which permits any noncommercial use, distribution, and reproduction in any medium, provided the original author(s) and source are credited.

\section{References}

1. Avron, J.E., Elgart, A., Graf, G.M., Sadun, L.: Transport and dissipation in quantum pumps. J. Stat. Phys. 116, 425-473 (2004)

2. Beenakker, C.W.J.: Random matrix theory of quantum transport. Rev. Mod. Phys. 69, 731 (1997)

3. Beenakker, C.W.J., Rejaei, B.: Nonlogarithmic repulsion of transmission eigenvalues in a disordered wire. Phys. Rev. Lett. 71, 3689-3692 (1993)

4. Caselle, M.: Distribution of transmission eigenvalues in disordered wires. Phys. Rev. Lett. 74, 2776-2779 (1995)

5. Caselle, M., Magnea, U.: Random matrix theory and symmetric spaces. Phys. Rep. 394, 41-156 (2004)

6. Dorokhov, O.N.: JETP Lett. 36, 318 (1982)

7. Dorokhov, O.N.: Solvable model of multichannel localization. Phys. Rev. B 37(18), 10526 (1988)

8. Dyson, F.: A Brownian-motion model for the eigenvalues of a random matrix. J. Math. Phys. 3, 1191 (1962)

9. Efetov, K.B., Larkin, A.I.: Zh. Eksp. Teor. Fiz. 85, 764 (1983)

10. Erdös, L., Yau, H.-T.: Linear Boltzmann equation as the weak coupling limit of a random Schrödinger equation. Commun. Pure Appl. Math. 53(6), 667-735 (2000)

11. Erdös, L., Peche, S., Ramirez, J.A., Schlein, B., Yau, H.T.: Bulk universality for Wigner matrices. Preprint arXiv:0905.4176

12. Erdös, L., Salmhofer, M., Yau, H.-T.: Quantum diffusion of the random Schrödinger evolution in the scaling limit i. the non-recollision diagrams. Acta Math. 200, 211-277 (2008) 
13. Erdös, L., Schlein, B., Yau, H.-T.: Universality of random matrices and local relaxation flow. Preprint arXiv:0907.5605

14. Ethier, S.N., Kurtz, T.G.: Markov Processes: Characterization and Convergence. Wiley Series in Probability and Statistics. Wiley, New York (1986)

15. Fouque, J.-P., Garnier, J.J., Papanicolaou, G., Solna, K.: Wave Propagation and Time Reversal in Randomly Layered Media. Wave Propagation and Time Reversal in Randomly Layered Media, vol. 56. Springer, Berlin (2007)

16. Gertsenshtein, M.E., Vasil'ev, V.B.: Waveguide with random non-homogeneities and Brownian motion on the Lobachevskii plane. Theor. Probab. Appl. 4, 391-398 (1959)

17. Guionnet, A.: Large Random Matrices: Lectures on Macroscopic Asymptotics. Lecture Notes in Mathematics. vol. 1957. Springer, Berlin (2006). Ecole d'Eté Probabilité. Saint-Flour XXXVI

18. Hüffmann, A.: Disordered wires from a geometric viewpoint. J. Phys. A: Math. Gen. 23, 5733-5744 (1990)

19. Mello, P.A., Pereyra, P., Kumar, N.: Ann. Phys. 181(2), 290-317 (1988)

20. Øksendal, B.: Stochastic Differential Equations, 6th edn. Universitext. Springer, Berlin (2003)

21. Reed, M., Simon, B.: Methods of Modern Mathematical Physics III. Academic Press, New York (1972)

22. Sadel, C., Schulz-Baldes, H.: Random Lie group actions on compact manifolds: a perturbative analysis. Preprint arXiv:0802.2909 (2008)

23. Schulz-Baldes, H.: Perturbation theory for Lyapunov exponents of an Anderson model on a strip. J. Geom. Funct. Anal. 14(5), 1089-1117 (2004)

24. Schulz-Baldes, H., Römer, R.: The random phase property and the Lyapunov spectrum for disordered mutli-channel systems

25. Spohn, H.: Derivation of the transport equation for electrons moving through random impurities. J. Stat. Phys. 17, 385-412 (1977)

26. Stroock, D.W., Varadhan, S.R.S.: Multidimensional Diffusion Processes. Grundlehren der Mathematischen Wissenschaften, vol. 233. Springer, Berlin (1979)

27. Valko, B., Virag, B.: Continuum limits of random matrices and the Brownian carousel. Invent. Math. 177, 463-508 (2009)

28. Valko, B., Virag, B.: Random Schrodinger operators on long boxes, noise explosion and the GOE. Preprint arXiv:0912.0097 (2009)

29. Wegner, F.J.: Disordered system with $n$ orbitals per site: $n=\infty$ limit. Phys. Rev. B 19(2), 783-792 (1979)

30. Zirnbauer, M.: Super Fourier analysis and localization in disordered wires. Phys. Rev. Lett. 69, 15841587 (1992) 